Article

\title{
Short-Term Effects of Organic Amendments on Soil Properties and Maize (Zea maize L.) Growth
}

\author{
Alessandro Calamai ${ }^{1}$, David Chiaramonti ${ }^{2,3}{ }^{,}$David Casini ${ }^{2}$, Alberto Masoni ${ }^{1}$ and \\ Enrico Palchetti ${ }^{1, *}$ (iD \\ 1 DAGRI, Department of Agriculture, Food, Environment and Forestry, University of Florence, \\ Piazzale delle Cascine 18, 50144 Firenze, Italy; Alessandro.calamai@unifi.it (A.C.); \\ alberto.masoni@unifi.it (A.M.) \\ 2 RE-CORD, Renewable Energy Consortium for R\&D, Viale J. F. Kennedy, 182, 50038 Scarperia e San Piero, \\ Italy; David.chiaramonti@polito.it (D.C.); david.casini@re-cord.org (D.C.) \\ 3 POLITO, Polytechnic of Turin, Corso Duca degli Abruzzi 24, 10129 Turin, Italy \\ * Correspondence: enrico.palchetti@unifi.it; Tel.: +39-055-2755800
}

Received: 27 February 2020; Accepted: 30 April 2020; Published: 8 May 2020

\begin{abstract}
In recent years, the application of biochar as soil amendment has generated a huge interest for the preservation of soil fertility by improving the physicochemical and biological properties of soil, and for the reduction of the negative effects of greenhouse emissions (climate-change adaptation). In this study, we investigated the effect of three soil amendments, namely, biochars derived from wood (BC), solid digestate (SD), and biochar derived from solid digestate (BSD), on soil parameters and their influence in maize-growth performance. The experiment was conducted in a greenhouse where organic amendments were applied to the soil control $(\mathrm{C})$ at different application rates: $0 \%, 1 \%$, $2 \%$, and $3 \% \mathrm{w} / \mathrm{w}$ (equivalent at $0,10,20$, and $30 \mathrm{t} \mathrm{ha}^{-1}$, respectively). The results indicated that all applications of organic amendments significantly enhanced soil parameters such as $\mathrm{pH}$ and electrical conductivity, while only BSD and SD showed a significant increase in secondary macro-(i.e., Ca, $\mathrm{Mg}$ ) and micronutrient-element content (i.e., $\mathrm{Na}, \mathrm{Fe}, \mathrm{Mn}$ and $\mathrm{Zn}$ ). The best maize performance (i.e., plant height, stem diameter, biomass dry weight, seed number, and weight per ear) was observed while using BSD, while plant growth in BC had reduced results, probably due to the low presence of elemental content and the relatively high volatile-matter (VM) content, which may have altered nitrogen dynamics.
\end{abstract}

Keywords: wood-derived biochar; nutrient-rich biochar; solid digestate; maize performance; organic amendment; soil management

\section{Introduction}

Biochar is a solid black carbon-rich material obtained by the thermochemical carbonization of lignocellulosic biomass in an oxygen-depleted atmosphere [1,2]. By selecting a reactor type that is suitable for each specific material, it can be produced from a wide range of feedstock (e.g., agriculture residues, green waste, animal manure, wood, and bark) using different thermal-process technologies (in particular, slow pyrolysis and gasification) that differ by maximal process temperature, heating rate, and residence time [3,4]. Feedstock characteristics, reactor configuration, and process parameters affect biochar yield and main physicochemical characteristics, such as pore structure, surface area, $\mathrm{pH}$, electrical conductivity (EC), and nutrient content, as reported by several authors [5-7].

Biochar application in soil is increasingly being recognized as a promising method to mitigate the negative effects of greenhouse-gas carbon dioxide $\left(\mathrm{CO}_{2}\right)$ through safe and long-term carbon sequestration while reducing methane $\left(\mathrm{CH}_{4}\right)$ and nitrous oxide $\left(\mathrm{N}_{2} \mathrm{O}\right)$ emissions in the atmosphere $[8,9]$. 
This sustainable approach is promoted by the stable nature, chemical recalcitrance, and resistance to biological degradation of biochar, which ensures a slowly cycling reservoir for at least one hundred years $[1,6]$. Moreover, the use of biochar as soil amendment seems to preserve soil fertility by improving the physicochemical and biological properties of soil, for example, liming $\mathrm{pH}$, promoting bacterium and fungus propagation, supporting soil structure, increasing cation exchange capacity (CEC), and ameliorating water-holding capacity and nutrient availability $[1,3,7]$. In particular, the authors suggested that biochar produced from nutrient-rich feedstock such as digestate possesses higher nutrient contents than that of biochar obtained from nutrient-poor feedstock (i.e., wood residues), which determines lower soil benefits due to lower mineralization rates $[9,10]$. From a plant-growth standpoint, open-field or pot biochar application exerts highly variable results, reporting a positive, minimal, or negative influence [9,11,12]. Contrasting results for different biochars were observed in sandy-loam soils. For instance, Butnan et al. [13] reported that biochar positively affected maize growth, and also persistent positive (liming) and negative (cation antagonism) effects in soil, whereas Nelissen et al. [14] showed little effects of biochar on soil quality, crop yield, and nutrient uptake. The use of biochar was demonstrated to positively affect crop yield on only some specific marginal soil, and this suggests that the relationship between biochar, soil-nutrient dynamics, plant roots, and micro-organisms in the rhizosphere is still far from being fully understood $[15,16]$. This could be due to the following factors: (i) even in the same pyrolysis-process conditions, physical and chemical biochar properties may vary depending on different feedstocks; (ii) the use of different rates and methods for soil incorporation; (iii) the presence of potentially toxic compounds (i.e., heavy metals) in the initial biomass, or polycyclic aromatic hydrocarbons (PAHs) and dioxins formed during the thermochemical-conversion process [16-19], which, in turn, depends on how biochar is produced and extracted from the reactor; and (iv) type of soil (this being probably the most relevant element among the other factors listed here).

Ongoing studies suggest a different effect on soil parameters and crop-yield improvement using wood-derived biochar or nutrient-rich derived biochar [20-22]. On this basis, the objectives of this study were: (i) characterize three different organic soil amendments, namely, biochars derived from woodchips (BC), solid digestate (SD), and biochar derived from solid digestate (BSD); (ii) determine the major effects on soil properties by amendment addition; and (iii) as a result, evaluate the amendment-induced influence on Zea maize L. growth.

\section{Materials and Methods}

\subsection{Experiment-Material Description}

Experiment soil was collected from $0-15 \mathrm{~cm}$ depth at the Istituto Tecnico Agrario, Florence, Italy $\left(43^{\circ} 47^{\prime} 07^{\prime \prime} \mathrm{N} ; 11^{\circ} 13^{\prime} 07^{\prime \prime} \mathrm{W}\right)$, air-dried, and thoroughly mixed, after which soil texture was determined. Fresh digestate was collected from a mesophilic anaerobic-digestion plant located in Grosseto (Italy) fed with agroindustrial residues and herbaceous biomass. Solid digestate was obtained by drying the digestate as received according to UNI EN 13040.

Biochars were produced from two different types of feedstock (solid digestate and woodchips) by slow pyrolysis using two different pilot units (Figure 1). Experiment apparatus PyroPilot Unit that was utilized for producing biochar from solid digestate (BSD) through a slow-pyrolysis process consists of a stirred tubular stainless-steel reactor of about $30 \mathrm{~L}$ inner volume, equipped with a double counterflow water-condensing system for the continuous extraction of pyrogases. Reactor heating was achieved by employing an electric heater, and the inert atmosphere was ensured by constant nitrogen flow. Carbonization was carried out at the maximal process temperature of $500^{\circ} \mathrm{C}$, reached with a heating rate of $25^{\circ} \mathrm{C} \mathrm{min}{ }^{-1}$. Feedstock was held in this pyrolysis condition for $3 \mathrm{~h}$, continuously mixed by rotating paddles. At the end of the set reaction time, the reactor was left overnight to cool down to ambient temperature (while pyrogas extraction continued), and the biochar was then collected from the reactor. 


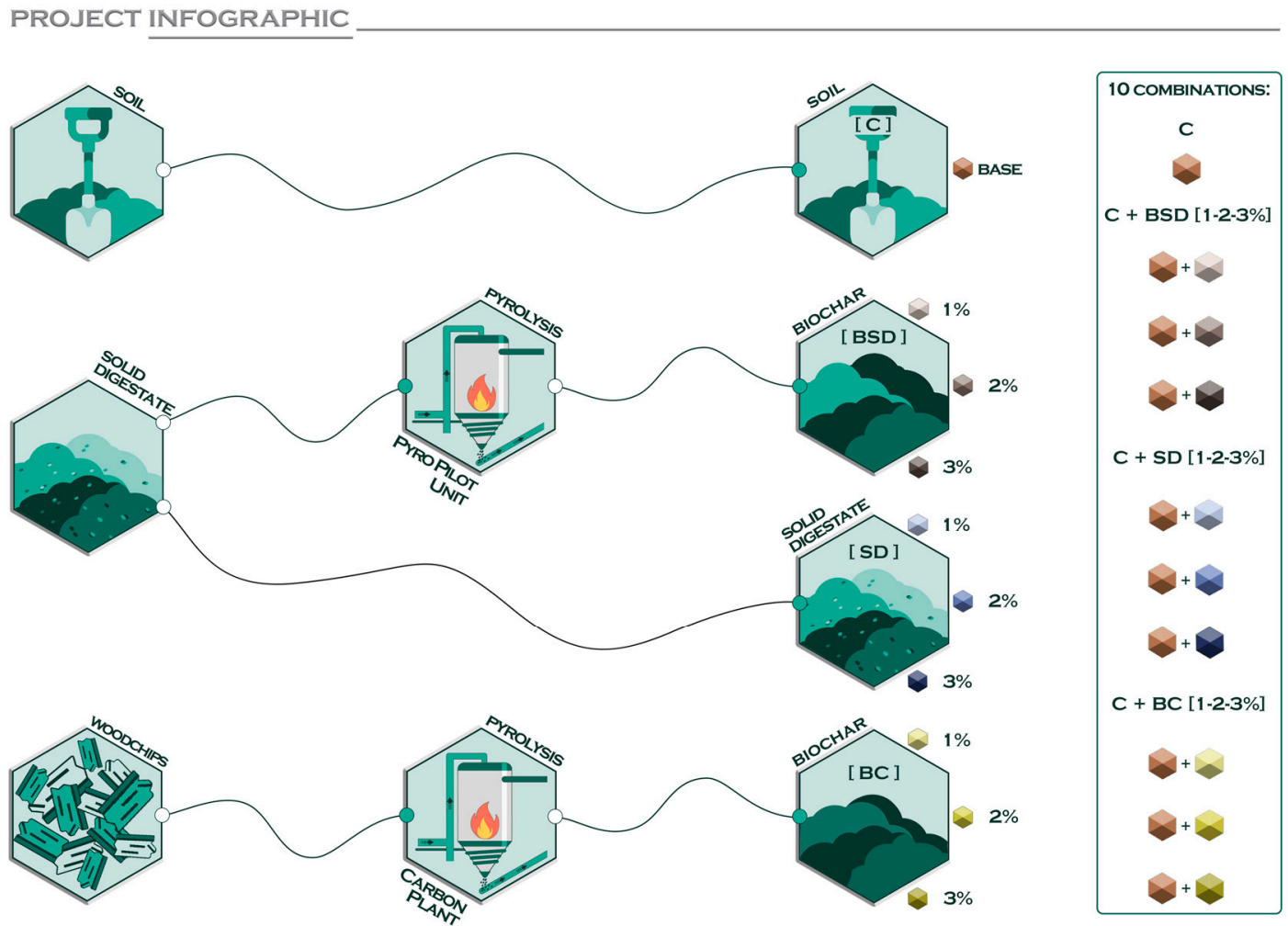

Figure 1. Experiment design.

Biochar from woodchips (BC) was produced in the oxidative CarbOn pilot plant developed by RE-CORD. The plant consists of a continuous biomass-carbonization system based on open-top downdraft technology. The cylindrical reactor (externally insulated) operates under an oxidative-pyrolysis regime, where biomass (with up to $20 \% \mathrm{w} / \mathrm{w}$ moisture content at inlet) was converted in a controlled oxidative environment. A more detailed description of the process and the pilot plant can be found in literature [23]. During the biochar-production test, the plant was fed with $50 \mathrm{~kg} \mathrm{~h}^{-1}$ of chestnut (Castanea sativa Mill.) woodchips. The maximal process temperature was about $550^{\circ} \mathrm{C}$, with a solid residence time of approximately 3 hours in the reactor.

Prior to laboratory analysis, samples (three replicates) of each experiment component were dried at $30-35^{\circ} \mathrm{C}$ for 6 days, ground in a ceramic mortar, and sifted through a $2 \mathrm{~mm}$ sieve.

In setting up the experiment trial, the $\mathrm{pH}$, electrical conductivity (EC), macronutrients, micronutrients, and heavy metals in soil, amendments, and each experiment treatment were determined. Total C, H, N, and S contents were analyzed using a CHNS analyzer (LECO Corp. St. Joseph, MI, USA) following ASTM method D5373, while P content was determined following the Olsen and Sommers [24] method. Mineral-fraction content ( $\mathrm{Ca}, \mathrm{Cd}, \mathrm{Cr}, \mathrm{Cu}, \mathrm{Fe}, \mathrm{K}, \mathrm{Mg}, \mathrm{Mn}, \mathrm{Na}, \mathrm{Pb}$, and $\mathrm{Zn}$ ) was detected using an inductively coupled plasma optical emission spectrometer (ICP, model IRIS Intrepid II XSP Radial, Thermo Fisher Scientific, Waltham, MA, USA) according to EPA method SW6020. EC and $\mathrm{pH}$ were determined in water extracts (sample: deionized water ratio of $1: 1, \mathrm{w} / \mathrm{w}$ ) by employing a portable EC meter (Hanna Instruments HI 9313-6) and a pH meter (pHenomenal pH 1100L) following the method previously described by Ahmedna et al. [25]. Proximate analysis on biochars, including volatile matter (VM), ash content, fixed carbon content (fixed C), was performed according to ASTM 1762-84 (Table 1). 
Table 1. Characteristics of soil amendments tested in maize cultivation.

\begin{tabular}{|c|c|c|c|c|}
\hline & & \multicolumn{3}{|c|}{ Treatment } \\
\hline & & BSD & SD & BC \\
\hline $\mathrm{pH}$ & & $8.48 \pm 0.10$ & $7.12 \pm 0.07$ & $8.17 \pm 0.06$ \\
\hline EC & & $6.86 \pm 0.12$ & $6.21 \pm 0.06$ & $6.49 \pm 0.14$ \\
\hline Fixed C & \multirow{3}{*}{$\%$} & $45.39 \pm 0.95$ & NA & $60.13 \pm 1.27$ \\
\hline Ash & & $38.82 \pm 0.61$ & NA & $2.75 \pm 0.04$ \\
\hline VM & & $15.79 \pm 0.18$ & NA & $37.12 \pm 0.68$ \\
\hline $\mathrm{C}$ & \multirow{9}{*}{$\mathrm{g} / \mathrm{kg}$} & $521.66 \pm 3.76$ & $385.78 \pm 3.30$ & $731.57 \pm 4.66$ \\
\hline $\mathrm{N}$ & & $13.85 \pm 0.36$ & $12.15 \pm 0.28$ & $7.51 \pm 0.19$ \\
\hline $\mathrm{P}$ & & $18.16 \pm 0.42$ & $8.08 \pm 0.20$ & $5.75 \pm 0.15$ \\
\hline $\mathrm{K}$ & & $28.32 \pm 0.45$ & $13.72 \pm 0.16$ & $9.53 \pm 0.09$ \\
\hline $\mathrm{Ca}$ & & $39.19 \pm 0.57$ & $22.05 \pm 0.26$ & $17.98 \pm 0.22$ \\
\hline $\mathrm{Mg}$ & & $8.53 \pm 0.25$ & $3.78 \pm 0.16$ & $4.38 \pm 0.08$ \\
\hline $\mathrm{Na}$ & & $1.31 \pm 0.07$ & $0.93 \pm 0.04$ & $0.94 \pm 0.01$ \\
\hline $\mathrm{S}$ & & ND & $2.93 \pm 0.04$ & $0.78 \pm 0.03$ \\
\hline $\mathrm{Fe}$ & & $1.93 \pm 0.03$ & $0.99 \pm 0.03$ & $1.27 \pm 0.05$ \\
\hline $\mathrm{Pb}$ & \multirow{6}{*}{$\mathrm{mg} / \mathrm{kg}$} & $1.07 \pm 0.04$ & $1.32 \pm 0.13$ & $1.29 \pm 0.04$ \\
\hline Mn & & $444.61 \pm 4.26$ & $160.73 \pm 3.45$ & $152.73 \pm 2.63$ \\
\hline $\mathrm{Cu}$ & & $64.75 \pm 1.76$ & $35.07 \pm 1.26$ & $43.79 \pm 1.03$ \\
\hline $\mathrm{Zn}$ & & $387.2 \pm 4.34$ & $176.1 \pm 2.60$ & $225.4 \pm 2.55$ \\
\hline $\mathrm{Cr}$ & & $14.15 \pm 0.42$ & $7.53 \pm 0.25$ & $9.53 \pm 0.47$ \\
\hline $\mathrm{Cd}$ & & $0.28 \pm 0.01$ & $0.05 \pm 0.00$ & $0.54 \pm 0.04$ \\
\hline
\end{tabular}

Means values of three replicates \pm standard error. BSD, solid-digestate-derived biochar; SD, solid digestate; $\mathrm{BC}$, wood-derived biochar; NA, not available; ND, not detectable.

\subsection{Experiment Design}

The experiment was conducted in a greenhouse tunnel located at the University of Florence, Department of Agriculture, Food, Environment, and Forestry (DAGRI) using cylindric pots of $5000 \mathrm{~cm}^{3}$ volume each (diameter of $0.20 \mathrm{~m}$ ) filled with the following substrates (Figure 1): 100\% soil as control (C), soil with two biochar types (BSD and BC) and solid digestate (SD) at different rates of amendment application, $1 \%, 2 \%$, and $3 \% \mathrm{w} / \mathrm{w}$ (equivalent to 10,20 , and $30 \mathrm{tha}^{-1}$, respectively; Table 2 ). No inorganic fertilizer was distributed. The experiment was laid out in a randomized complete block design (RCBD) with seven replications for each substrate (a total of 70 pots). Seeds of a commercial maize hybrid (Belgrano, Limagrain, FAO 300) were used as test crops. During the growing season (May-August), the average temperature recorded inside the greenhouse tunnel was $29.3^{\circ} \mathrm{C}$, with the average minimal air temperatures found in May $\left(19.2^{\circ} \mathrm{C}\right)$, and the average maximum $\left(35.4^{\circ} \mathrm{C}\right)$ in July. Within each treatment, four pots were randomly selected and equipped with a soil-moisture sensor. Moreover, a scheduled automatic-irrigation program was set up to maintain soil-moisture content at $60 \%$, thus avoiding water stress. 
Table 2. Chemical properties of soil (C) and soil after amendment addition in maize cultivation \pm standard error.

\begin{tabular}{|c|c|c|c|c|c|c|c|c|c|c|c|c|c|c|c|c|}
\hline \multirow[b]{2}{*}{ Treatment } & \multirow[b]{2}{*}{$\mathrm{pH}$} & \multirow[b]{2}{*}{ EC } & \multicolumn{8}{|c|}{$\mathrm{g} / \mathrm{kg}$} & \multicolumn{6}{|c|}{$\mathrm{mg} / \mathrm{kg}$} \\
\hline & & & $\mathbf{N}$ & $P$ & K & $\mathrm{Ca}$ & $\mathrm{Mg}$ & $\mathrm{Na}$ & $\mathrm{S}$ & $\mathrm{Fe}$ & $\mathrm{Pb}$ & Mn & $\mathrm{Cu}$ & $\mathrm{Zn}$ & $\mathrm{Cr}$ & $\mathrm{Cd}$ \\
\hline $\mathrm{C}+\mathrm{BSD} 1 \%$ & $7.25 \pm 0.04 a b$ & $0.59 \pm 0.01 \mathrm{a}$ & $1.79 \pm 0.09$ & $0.93 \pm 0.04$ & $7.75 \pm 0.08$ & $1.59 \pm 0.01 \mathrm{~d}$ & $0.54 \pm 0.02 b$ & $0.52 \pm 0.01 \mathrm{~b}$ & $1.95 \pm 0.07$ & $3.15 \pm 0.03 \mathrm{~b}$ & $1.45 \pm 0.09$ & $53.47 \pm 0.54 b$ & $73.94 \pm 2.92$ & $304.86 \pm 3.11 \mathrm{~b}$ & $12.67 \pm 0.80$ & $5.43 \pm 1.07$ \\
\hline $\mathrm{C}+\mathrm{BSD} 2 \%$ & $7.29 \pm 0.01 \mathrm{a}$ & $0.63 \pm 0.02 a$ & $1.91 \pm 0.07$ & $0.91 \pm 0.05$ & $8.04 \pm 0.03$ & $1.78 \pm 0.03 \mathrm{~b}$ & $0.63 \pm 0.01 \mathrm{a}$ & $0.54 \pm 0.02 \mathrm{~b}$ & $1.72 \pm 0.06$ & $3.28 \pm 0.03 \mathrm{ab}$ & $0.97 \pm 0.08$ & $52.83 \pm 0.74 \mathrm{~b}$ & $75.34 \pm 5.30$ & $313.23 \pm 2.77 \mathrm{~b}$ & $13.59 \pm 0.54$ & $4.79 \pm 1.16$ \\
\hline $\mathrm{C}+\mathrm{BSD} 3 \%$ & $7.32 \pm 0.03 a$ & $0.61 \pm 0.02 a$ & $2.02 \pm 0.10$ & $1.04 \pm 0.05$ & $7.98 \pm 0.08$ & $1.93 \pm 0.02 \mathrm{a}$ & $0.61 \pm 0.01 \mathrm{a}$ & $0.69 \pm 0.01 \mathrm{a}$ & $2.08 \pm 0.04$ & $3.35 \pm 0.02 a$ & $1.61 \pm 0.06$ & $57.91 \pm 0.84 \mathrm{a}$ & $63.47 \pm 2.73$ & $354.74 \pm 3.04 a$ & $13.28 \pm 0.53$ & $5.67 \pm 0.54$ \\
\hline C + SD $1 \%$ & $6.96 \pm 0.02 \mathrm{~d}$ & $0.41 \pm 0.02 \mathrm{c}$ & $1.95 \pm 0.08$ & $0.88 \pm 0.03$ & $7.84 \pm 0.06$ & $1.52 \pm 0.02 \mathrm{~d}$ & $0.49 \pm 0.01 \mathrm{c}$ & $0.41 \pm 0.01 \mathrm{~d}$ & $1.65 \pm 0.08$ & $2.75 \pm 0.02 \mathrm{~d}$ & $1.64 \pm 0.08$ & $49.63 \pm 1.48 \mathrm{~d}$ & $58.26 \pm 2.96$ & $263.69 \pm 5.38 c$ & $13.68 \pm 0.69$ & $5.16 \pm 0.61$ \\
\hline$C+S D 2 \%$ & $7.06 \pm 0.00 c$ & $0.43 \pm 0.01 \mathrm{c}$ & $1.88 \pm 0.08$ & $0.95 \pm 0.06$ & $7.64 \pm 0.06$ & $1.62 \pm 0.02 \mathrm{~d}$ & $0.50 \pm 0.00 \mathrm{c}$ & $0.44 \pm 0.01 \mathrm{~cd}$ & $1.85 \pm 0.08$ & $2.93 \pm 0.02 c$ & $0.81 \pm 0.05$ & $50.17 \pm 1.17 \mathrm{~cd}$ & $32.37 \pm 2.83$ & $275.32 \pm 4.16 \mathrm{c}$ & $12.98 \pm 0.66$ & $6.87 \pm 0.57$ \\
\hline$C+$ SD $3 \%$ & $7.03 \pm 0.01 \mathrm{c}$ & $0.48 \pm 0.03 \mathrm{bc}$ & $1.98 \pm 0.06$ & $0.87 \pm 0.04$ & $7.79 \pm 0.04$ & $1.68 \pm 0.01 \mathrm{c}$ & $0.53 \pm 0.01 \mathrm{~b}$ & $0.46 \pm 0.01 \mathrm{~cd}$ & $2.53 \pm 0.09$ & $2.95 \pm 0.02 c$ & $1.13 \pm 0.06$ & $51.29 \pm 1.07 \mathrm{c}$ & $77.43 \pm 3.07$ & $288.96 \pm 5.01 \mathrm{~b}$ & $11.87 \pm 0.73$ & $4.56 \pm 0.34$ \\
\hline$C+B C 1 \%$ & $7.21 \pm 0.01 \mathrm{~b}$ & $0.49 \pm 0.02 \mathrm{bc}$ & $1.52 \pm 0.12$ & $0.97 \pm 0.04$ & $7.96 \pm 0.06$ & $1.61 \pm 0.01 \mathrm{~d}$ & $0.51 \pm 0.02 \mathrm{bc}$ & $0.49 \pm 0.02 b c$ & $2.14 \pm 0.09$ & $2.87 \pm 0.03 \mathrm{~cd}$ & $1.39 \pm 0.08$ & $47.49 \pm 1.59 \mathrm{e}$ & $32.74 \pm 4.69$ & $189.76 \pm 4.89 \mathrm{~d}$ & $11.57 \pm 0.88$ & $5.54 \pm 0.39$ \\
\hline$C+B C 2 \%$ & $7.19 \pm 0.01 \mathrm{~b}$ & $0.51 \pm 0.01 \mathrm{~b}$ & $1.75 \pm 0.07$ & $0.88 \pm 0.04$ & $7.83 \pm 0.05$ & $1.70 \pm 0.04 \mathrm{c}$ & $0.65 \pm 0.01 \mathrm{a}$ & $0.47 \pm 0.01 \mathrm{c}$ & $2.37 \pm 0.08$ & $3.11 \pm 0.01 \mathrm{~b}$ & $0.57 \pm 0.09$ & $48.43 \pm 1.72 \mathrm{e}$ & $41.93 \pm 2.06$ & $232.31 \pm 5.58 \mathrm{~d}$ & $13.06 \pm 0.77$ & $6.91 \pm 1.14$ \\
\hline$C+B C 3 \%$ & $7.27 \pm 0.04 a b$ & $0.53 \pm 0.02 b$ & $1.78 \pm 0.08$ & $0.98 \pm 0.04$ & $8.04 \pm 0.12$ & $1.73 \pm 0.03 \mathrm{c}$ & $0.64 \pm 0.01 \mathrm{a}$ & $0.51 \pm 0.01 \mathrm{~b}$ & $2.43 \pm 0.08$ & $3.08 \pm 0.02 b$ & $1.43 \pm 0.08$ & $48.67 \pm 0.95 \mathrm{e}$ & $98.45 \pm 4.74$ & $239.87 \pm 4.09 \mathrm{c}$ & $14.94 \pm 0.91$ & $5.64+1.09$ \\
\hline C & $7.05 \pm 0.01 \mathrm{c}$ & $0.45 \pm 0.01 \mathrm{c}$ & $1.69 \pm 0.06$ & $1.01 \pm 0.03$ & $7.91 \pm 0.10$ & $1.54 \pm 0.02 \mathrm{e}$ & $0.28 \pm 0.01 \mathrm{~d}$ & $0.38 \pm 0.00 \mathrm{e}$ & $2.13 \pm 0.06$ & $2.76 \pm 0.01 \mathrm{~d}$ & $0.36 \pm 0.05$ & $13.52 \pm 0.63 \mathrm{f}$ & $65.18 \pm 2.53$ & $142.52 \pm 5.22 \mathrm{e}$ & $14.21 \pm 0.43$ & $5.47 \pm 0.83$ \\
\hline F-test & & & n.s & n.s & n.s & & & & n.s & & n.s & & n.s & & n.s & $\mathrm{n} . \mathrm{s}$ \\
\hline
\end{tabular}

BSD, solid-digestate-derived biochar; SD, solid digestate; BC, wood-derived biochar. Means followed by the same letters on the column are not significant at $p>0.05$, and means followed by different letters on the column are significant at $p<0.05$. F-test values correspond to different $\mathrm{p}$ values: “* $=p<0.05$, , $* * * \prime=p<0.001$, “n.s" $=p>0.05$. 


\subsection{Plant Sampling and Analysis}

The different maize phenological-development stages were monitored following the Biologische Bundesanstalt, Bundessortenamt und Chemische Industrie (BBCH) scale. Growing nondestructive parameters (i.e., plant height and diameter) were first collected 30 days after germination (DAG) starting at $\mathrm{BBCH}$ growth stage 13 (i.e., three leaves unfolded) until $\mathrm{BBCH}$ growth stage 69 (i.e., end of flowering) every ten days. The whole growing cycle was completed in about 90 days, and at harvest time some destructive parameters were detected on every single plant, such as plant height $(\mathrm{cm})$, stem diameter $(\mathrm{mm})$, biomass dry weight $(\mathrm{g})$, seed number per ear, and seed weight $(\mathrm{g})$. To determine tissue nutrient concentrations, three fully expanded and healthy leaves (top, middle, and bottom of plant height) were collected from each plant at harvest time, oven-dried at $55^{\circ} \mathrm{C}$ until constant weight was achieved, ground, sifted through a $2 \mathrm{~mm}$ sieve, and analyzed according to the CHNS and ICP methods.

\subsection{Statistical Analysis}

Statistical analysis was performed using R software [26]. The significant effects produced by amendment application on soil properties, maize vegetative and growth parameters, and plant-tissue nutrient content were assessed and compared using univariate analysis fitting a generalized linear model (GLM). All variables were separately analyzed using the R/lme4 package [27] and considered as the main factor amendment types. The effects of different amendment concentrations were compared using a post hoc test creating a dummy matrix of contrast with the mcp function that was then used as the $\mathrm{k}$ parameter in the glht function of the R/multcomp package [28], following the method of Hothorn et al. [29].

\section{Results}

\subsection{Soil, Amendments, and Treatment Properties}

The main chemical-physical properties of the tested amendments are reported in Table 1. Concerning $\mathrm{pH}$, biochars were characterized by alkaline value, with the highest recorded in BSD (8.48), followed by BC (8.17), while neutral $\mathrm{pH}$ (7.12) was reported for SD. At the same time, BSD showed a higher EC value $\left(6.86 \mathrm{mS} \mathrm{cm}^{-1}\right)$ than $\mathrm{BC}\left(6.49 \mathrm{mS} \mathrm{cm}^{-1}\right)$ and $\mathrm{SD}\left(6.21 \mathrm{mS} \mathrm{cm}^{-1}\right)$. Proximate analysis indicated that wood-derived biochar $(\mathrm{BC})$ had the highest fixed $\mathrm{C}$ and $\mathrm{VM}$ content $(60.13$ and $37.12 \%$, respectively), but the lowest ash content (2.75\%). Nutrient-rich biochar (BSD), on the other hand, showed higher ash content (38.82\%), but the lowest fixed C and VM content (45.39 and 15.79\%, respectively). Between the selected amendments, BC showed greater total $\mathrm{C}$ concentration $\left(731.57 \mathrm{~g} \mathrm{~kg}^{-1}\right)$ compared to BSD and SD (521.68 and $385.78 \mathrm{~g} \mathrm{~kg}^{-1}$, respectively), while BSD showed the highest macro- and microelement concentration, with the only exception for $\mathrm{S}$ content, which was absent. Unamended experimental soil (C) properties are reported in Table 2; it was characterized by a sandy-loam texture (56.78\% sand, $29.15 \%$ silt, and $14.07 \%$ clay), neutral $\mathrm{pH}$ (7.05), and low EC $\left(0.45 \mathrm{mS} \mathrm{cm}^{-1}\right)$.

The addition of BSD, BC, and SD induced several changes in soil chemical characteristics and nutrient concentrations in the tested treatments (Table 2). The amendment addition significantly affected $\mathrm{pH}$ and EC values ( $p<0.05$ and $p<0.01$, respectively). In general, BC and BSD applications at increasing rates both led to the largest increment in both parameters, while SD application had no influence on $\mathrm{pH}$, and seemed to only increase EC value at 3\% of application level. At the same time, the addition of BC, BSD, and SD caused significant variability in soil nutrient contents, in particular, $\mathrm{Na}, \mathrm{Fe}$, and $\mathrm{Mn}$ contents showed high statistical significance $(p<0.01)$, while $\mathrm{Ca}$, $\mathrm{Mg}$, and $\mathrm{Zn}$ contents were less influenced by amendment application $(p<0.05)$. No statistical significance $(p>0.05)$ was found for detected macronutrient $(\mathrm{N}, \mathrm{P}$, and $\mathrm{K}), \mathrm{S}$, and heavy-metal contents $(\mathrm{Pb}, \mathrm{Cu}, \mathrm{Cr}$, and $\mathrm{Cd})$. In general, $\mathrm{SD}$ application to soil appeared to ameliorate nutrient contents, although less so than for BSD. However, these increments were proportional to the increasing application rates in both treatments. In contrast, BC application appeared to affect soil-nutrient concentrations less. 


\subsection{Plant Responses and Tissue Nutrient Concentration}

Significant differences between the treatments were observed in the detected maize vegetative parameters (Table 3). Overall, plant height, stem diameter, and biomass dry weight showed high statistical significance $(p<0.01)$, while seed number and seed weight per ear were less influenced by amendment application $(p<0.05)$. The maize plant cultivated in all substrates with an increasing $(1 \%, 2 \%$, and $3 \%)$ BC application rate showed the lowest value on biomass production (from 92.07 to $95.77 \mathrm{~g}$ ), seed number (from 163.71 to 169.72), and seed weight (from 34.19 to $35.59 \mathrm{~g}$ ). At the same time, using $\mathrm{C}+\mathrm{BC} 1 \%$ and $\mathrm{C}+\mathrm{BC} 2 \%$, plants showed the worst value on plant height $(68.14$ and $69.83 \mathrm{~cm}$, respectively) and stem diameter (11.75 and $12.14 \mathrm{~mm}$, respectively). $\mathrm{C}+\mathrm{BSD} 3 \%$ treatment increased all investigated vegetative parameters, reporting the highest seed weight $(56.20 \mathrm{~g})$, biomass production $(121.63 \mathrm{~g})$, and stem diameter $(16.53 \mathrm{~mm})$. The C + BSD2\% and C + BSD3\% treatments gave the highest value on plant height $(96.00$ and $99.13 \mathrm{~cm}$ ) and seed number $(211.86$ and 209.68, respectively). The $C+$ $\mathrm{SD} 1 \%$ and $\mathrm{C}+\mathrm{SD} 2 \%$ treatments showed vegetative parameters that were comparable with control. Only C + SD3\% seemed to improve biomass production $(+11 \%)$, seed number $(+9 \%)$, and seed weight $(+8 \%)$ with respect to the control.

Table 3. Effects of soil amendments on vegetative parameters in maize cultivation \pm standard error.

\begin{tabular}{cccccc}
\hline Treatment & $\begin{array}{c}\text { Plant Height } \\
\mathbf{( c m})\end{array}$ & $\begin{array}{c}\text { Stem Diameter } \\
(\mathbf{m m})\end{array}$ & $\begin{array}{c}\text { Biomass Dry } \\
\text { Weight } \mathbf{( g )}\end{array}$ & $\begin{array}{c}\text { Seed Number } \\
\text { per Ear }(\mathbf{n} .)\end{array}$ & $\begin{array}{c}\text { Seed Weight per } \\
\text { Ear } \mathbf{( g )}\end{array}$ \\
\hline C + BSD 1\% & $89.61 \pm 2.28 \mathrm{ab}$ & $15.44 \pm 0.16 \mathrm{~b}$ & $114.02 \pm 3.49 \mathrm{~b}$ & $198.29 \pm 3.27 \mathrm{~b}$ & $46.35 \pm 4.13 \mathrm{a}-\mathrm{c}$ \\
$\mathrm{C}+\mathrm{BSD} \%$ & $96.00 \pm 2.60 \mathrm{a}$ & $15.78 \pm 0.19 \mathrm{ab}$ & $110.42 \pm 4.53 \mathrm{~b}$ & $211.86 \pm 5.58 \mathrm{a}$ & $50.52 \pm 2.11 \mathrm{ab}$ \\
C + BSD3\% & $99.13 \pm 1.83 \mathrm{a}$ & $16.53 \pm 0.14 \mathrm{a}$ & $121.63 \pm 3.05 \mathrm{a}$ & $209.68 \pm 3.67 \mathrm{a}$ & $56.20 \pm 2.58 \mathrm{a}$ \\
C + SD 1\% & $81.45 \pm 1.34 \mathrm{c}$ & $13.31 \pm 0.21 \mathrm{c}-\mathrm{e}$ & $101.30 \pm 3.16 \mathrm{c}$ & $177.95 \pm 3.16 \mathrm{c}$ & $41.47 \pm 1.79 \mathrm{bc}$ \\
C + SD 2\% & $82.38 \pm 1.62 \mathrm{c}$ & $13.98 \pm 0.14 \mathrm{~b}-\mathrm{d}$ & $109.57 \pm 1.58 \mathrm{bc}$ & $183.43 \pm 2.52 \mathrm{bc}$ & $40.42 \pm 2.54 \mathrm{bc}$ \\
C + SD 3\% & $86.44 \pm 1.69 \mathrm{~b}$ & $14.33 \pm 0.27 \mathrm{bc}$ & $110.09 \pm 2.76 \mathrm{~b}$ & $192.86 \pm 4.49 \mathrm{~b}$ & $46.37 \pm 4.86 \mathrm{a}-\mathrm{c}$ \\
C + BC 1\% & $68.14 \pm 1.57 \mathrm{~d}$ & $11.75 \pm 0.13 \mathrm{e}$ & $92.07 \pm 3.28 \mathrm{~d}$ & $163.71 \pm 3.30 \mathrm{~d}$ & $35.59 \pm 3.44 \mathrm{c}$ \\
C + BC 2\% & $69.83 \pm 1.24 \mathrm{~d}$ & $12.14 \pm 0.09 \mathrm{e}$ & $93.59 \pm 0.42 \mathrm{~d}$ & $167.14 \pm 5.01 \mathrm{~d}$ & $34.19 \pm 3.90 \mathrm{c}$ \\
C + BC 3\% & $74.81 \pm 2.23 \mathrm{~cd}$ & $12.61 \pm 0.27 \mathrm{c}-\mathrm{e}$ & $95.77 \pm 0.34 \mathrm{~d}$ & $169.72 \pm 2.43 \mathrm{~d}$ & $35.25 \pm 2.50 \mathrm{c}$ \\
C & $76.33 \pm 1.35 \mathrm{~cd}$ & $12.42 \pm 0.17 \mathrm{~cd}$ & $97.88 \pm 0.10 \mathrm{~cd}$ & $174.51 \pm 2.27 \mathrm{c}$ & $42.59 \pm 2.32 \mathrm{bc}$ \\
\hline F-test & $* *$ & $* *$ & $* *$ & $*$ & $*$ \\
\hline
\end{tabular}

BSD, solid-digestate-derived biochar; SD, solid digestate; BC, wood-derived biochar; C, control. Means followed by the same letters on the column are not significant at $p>0.05$, and means followed by different letters on the column are significant at $p<0.05$. F-test values correspond to different $p$-values: " ${ }^{* \prime \prime}=p<0.05$, , ${ }^{\prime * * \prime \prime}=p<0.001$, "n.s" $=p>0.05$.

Significant differences $(p<0.01)$ were also recorded for both tested growth parameters (Table 4$)$. In general, from 30 to $90 \mathrm{DAG}$, treatments $\mathrm{C}+\mathrm{BSD} 2 \%$ and $\mathrm{C}+\mathrm{BSD} 3 \%$ had a similar effect, producing maximal height and stem diameter, while $\mathrm{C}+\mathrm{BC} 1 \%$ and $\mathrm{C}+\mathrm{BC} 2 \%$ showed the lowest values. At the same time, $\mathrm{C}+\mathrm{SD} 1 \%, \mathrm{C}+\mathrm{SD} 2 \%, \mathrm{C}+\mathrm{SD} 3 \%$, and $\mathrm{C}$ had similar values in both parameters up to 50 DAG; subsequently, plants cultivated in SD-added soils showed an increase in both parameters until the end of the experiment.

The application of different amendments significantly affected $\mathrm{Ca}, \mathrm{Mg}, \mathrm{Fe}(p<0.01), \mathrm{Na}$, and $\mathrm{Mn}$ $(p<0.05)$ accumulation in maize tissue (Table 5). Plants cultivated in C + BSD treatments showed the highest secondary macro- and micronutrient content ( $\mathrm{Ca}, \mathrm{Mg}, \mathrm{Fe}, \mathrm{Na}$, and $\mathrm{Mn}$ ), especially using $2 \%$ and $3 \%$ rates. Conversely, $\mathrm{C}+\mathrm{BC}$ treatments were reported to have the lowest plant nutrient concentrations, which were comparable with those of the untreated soil (C). 
Table 4. Effects of soil amendments on growth parameters in maize cultivation \pm standard error.

\begin{tabular}{|c|c|c|c|c|c|c|c|}
\hline \multirow[b]{2}{*}{ Treatment } & \multicolumn{7}{|c|}{ Plant-Height (cm) Evolution Over Ten Days } \\
\hline & $30 \mathrm{DAG}$ & 40 DAG & $50 \mathrm{DAG}$ & $60 \mathrm{DAG}$ & 70 DAG & 80 DAG & 90 DAG \\
\hline$C+B S D 1 \%$ & $14.25 \pm 0.56 \mathrm{~b}$ & $18.51 \pm 0.96 b$ & $27.53 \pm 1.11 \mathrm{a}-\mathrm{c}$ & $40.15 \pm 1.07 \mathrm{ab}$ & $49.84 \pm 2.20 \mathrm{a}-\mathrm{c}$ & $64.16 \pm 2.68 \mathrm{a}-\mathrm{c}$ & $89.61 \pm 2.28 \mathrm{ab}$ \\
\hline $\mathrm{C}+\mathrm{BSD} 2 \%$ & $14.79 \pm 0.77 \mathrm{ab}$ & $20.45 \pm 1.64 a$ & $28.72 \pm 0.60 \mathrm{ab}$ & $45.35 \pm 1.70 \mathrm{a}$ & $55.21 \pm 1.31 \mathrm{ab}$ & $68.23 \pm 1.77 \mathrm{ab}$ & $96.00 \pm 2.60 \mathrm{a}$ \\
\hline $\mathrm{C}+\mathrm{BSD} 3 \%$ & $15.45 \pm 0.74 \mathrm{a}$ & $21.57 \pm 0.98 \mathrm{a}$ & $30.87 \pm 0.64 \mathrm{a}$ & $46.58 \pm 1.40 \mathrm{a}$ & $56.83 \pm 1.19 a$ & $69.68 \pm 2.09 a$ & $99.13 \pm 1.83 a$ \\
\hline $\mathrm{C}+\mathrm{SD} 1 \%$ & $12.17 \pm 0.83 \mathrm{~cd}$ & $16.97 \pm 0.76 c-e$ & $22.94 \pm 1.16 \mathrm{~d}$ & $34.37 \pm 2.08 \mathrm{~b}-\mathrm{d}$ & $49.04 \pm 2.29 b-d$ & $58.14 \pm 2.22 c$ & $81.45 \pm 1.34 c$ \\
\hline$C+\operatorname{SD} 2 \%$ & $12.64 \pm 0.74 \mathrm{c}$ & $17.57 \pm 1.07 \mathrm{c}$ & $24.69 \pm 0.60 \mathrm{~cd}$ & $36.19 \pm 1.23 b c$ & $51.00 \pm 2.05 a-c$ & $62.13 \pm 1.61 b c$ & $82.38 \pm 1.62 c$ \\
\hline$C+\operatorname{SD} 3 \%$ & $12.57 \pm 1.20 c$ & $17.83 \pm 1.72 b c$ & $26.54 \pm 1.17 \mathrm{~b}-\mathrm{d}$ & $38.75 \pm 1.08 b$ & $53.42 \pm 2.22 a-c$ & $63.50 \pm 2.30 a-c$ & $86.44 \pm 1.69 \mathrm{~b}$ \\
\hline $\mathrm{C}+\mathrm{BC} 1 \%$ & $11.81 \pm 0.88 \mathrm{de}$ & $15.78 \pm 0.90 \mathrm{e}$ & $22.14 \pm 0.61 d$ & $27.31 \pm 0.87 \mathrm{~d}$ & $35.60 \pm 1.74 \mathrm{e}$ & $47.78 \pm 1.45 \mathrm{e}$ & $68.14 \pm 1.57 \mathrm{~d}$ \\
\hline$C+B C 2 \%$ & $11.25 \pm 1.01 \mathrm{e}$ & $15.98 \pm 0.99 \mathrm{e}$ & $23.02 \pm 0.92 \mathrm{~d}$ & $29.57 \pm 1.58 \mathrm{~d}$ & $36.73 \pm 1.12 \mathrm{e}$ & $48.37 \pm 2.05 \mathrm{e}$ & $69.83 \pm 1.24 \mathrm{~d}$ \\
\hline$C+B C 3 \%$ & $11.94 \pm 1.43 \mathrm{c}-\mathrm{e}$ & $16.75 \pm 1.09 \mathrm{de}$ & $24.02 \pm 0.69 \mathrm{~cd}$ & $31.95 \pm 1.14 \mathrm{~cd}$ & $37.47 \pm 1.56 \mathrm{de}$ & $51.36 \pm 2.68 \mathrm{de}$ & $74.81 \pm 2.23 \mathrm{~cd}$ \\
\hline C & $12.37 \pm 0.80 \mathrm{~cd}$ & $17.78 \pm 1.22 \mathrm{bc}$ & $25.19 \pm 1.01 c$ & $33.15 \pm 0.95 c$ & $43.15 \pm 1.50 \mathrm{de}$ & $55.23 \pm 1.36 \mathrm{~d}$ & $76.33 \pm 1.35 \mathrm{~cd}$ \\
\hline \multirow[t]{2}{*}{ F-test } & $* *$ & $* *$ & $* *$ & $* *$ & $* *$ & $* *$ & $* *$ \\
\hline & \multicolumn{7}{|c|}{ Stem-Diameter (mm) Evolution Over Ten Days } \\
\hline Treatment & 30 DAG & 40 DAG & 50 DAG & 60 DAG & 70 DAG & 80 DAG & 90 DAG \\
\hline $\mathrm{C}+\mathrm{BSD} 1 \%$ & $4.66 \pm 0.13 \mathrm{ab}$ & $6.51 \pm 0.20 \mathrm{a}-\mathrm{c}$ & $9.13 \pm 0.15 b$ & $11.45 \pm 0.16 b$ & $12.39 \pm 0.06 b$ & $13.71 \pm 0.14 b$ & $15.44 \pm 0.16 b$ \\
\hline $\mathrm{C}+\mathrm{BSD} 2 \%$ & $4.70 \pm 0.21 \mathrm{ab}$ & $7.49 \pm 0.16 \mathrm{ab}$ & $9.73 \pm 0.20 \mathrm{ab}$ & $11.75 \pm 0.17 \mathrm{ab}$ & $12.68 \pm 0.18 \mathrm{ab}$ & $14.49 \pm 0.19 \mathrm{ab}$ & $15.78 \pm 0.19 \mathrm{ab}$ \\
\hline $\mathrm{C}+\mathrm{BSD} 3 \%$ & $5.14 \pm 0.19 a$ & $7.95 \pm 0.16 a$ & $10.7 \pm 0.13 a$ & $12.89 \pm 0.16 \mathrm{a}$ & $13.38 \pm 0.12 \mathrm{a}$ & $14.76 \pm 0.21 \mathrm{a}$ & $16.53 \pm 0.14 a$ \\
\hline $\mathrm{C}+\mathrm{SD} 1 \%$ & $4.12 \pm 0.19 b c$ & $5.65 \pm 0.17 \mathrm{c}$ & $7.43 \pm 0.16 \mathrm{~d}$ & $10.42 \pm 0.20 c$ & $11.68 \pm 0.06 \mathrm{~b}-\mathrm{d}$ & $12.31 \pm 0.28 \mathrm{~b}-\mathrm{d}$ & $13.31 \pm 0.21 \mathrm{c}-\mathrm{e}$ \\
\hline$C+\operatorname{SD} 2 \%$ & $4.13 \pm 0.21 b c$ & $6.02 \pm 0.11 b c$ & $8.16 \pm 0.19 c d$ & $10.37 \pm 0.19 c$ & $11.65 \pm 0.23 b-d$ & $12.18 \pm 0.19 \mathrm{~cd}$ & $13.98 \pm 0.14 b-d$ \\
\hline$C+\operatorname{SD} 3 \%$ & $4.03 \pm 0.15 c$ & $6.35 \pm 0.13 b$ & $9.04 \pm 0.17 b$ & $10.68 \pm 0.16 c$ & $12.06 \pm 0.23 b c$ & $12.94 \pm 0.06 c$ & $14.33 \pm 0.27 b c$ \\
\hline$C+B C 1 \%$ & $3.77 \pm 0.11 \mathrm{~d}$ & $5.09 \pm 0.16 \mathrm{~d}$ & $7.32 \pm 0.15 d$ & $8.85 \pm 0.09 f$ & $9.98 \pm 0.11 d$ & $10.93 \pm 0.10 \mathrm{e}$ & $11.75 \pm 0.13 \mathrm{e}$ \\
\hline$C+B C 2 \%$ & $3.89 \pm 0.06 c$ & $5.82 \pm 0.15 b c$ & $7.55 \pm 0.09 \mathrm{~d}$ & $9.12 \pm 0.12 \mathrm{e}$ & $10.45 \pm 0.13 c d$ & $11.45 \pm 0.15 \mathrm{e}$ & $12.14 \pm 0.09 \mathrm{e}$ \\
\hline$C+B C 3 \%$ & $4.06 \pm 0.16 c$ & $5.91 \pm 0.16 b c$ & $7.48 \pm 0.12 d$ & $9.78 \pm 0.09 \mathrm{de}$ & $11.01 \pm 0.20 \mathrm{~cd}$ & $12.09 \pm 0.16 \mathrm{~d}$ & $12.61 \pm 0.27 c-e$ \\
\hline C & $4.28 \pm 0.13 b$ & $6.26 \pm 0.08 b$ & $8.47 \pm 0.11 c$ & $10.12 \pm 0.05 \mathrm{~cd}$ & $11.25 \pm 0.17 c$ & $11.98 \pm 0.18 \mathrm{~d}$ & $12.42 \pm 0.17 \mathrm{~cd}$ \\
\hline F-test & * & $* *$ & $* *$ & $* *$ & $* *$ & ** & ** \\
\hline
\end{tabular}

BSD, solid-digestate-derived biochar; $\mathrm{SD}$, solid digestate; $\mathrm{BC}$, wood-derived biochar; $\mathrm{C}$, control; $\mathrm{DAG}$, day after germination. Means followed by the same letters on the column are not significant at $\mathrm{P}>0.05$, and means followed by different letters on the column are significant at $\mathrm{P}<0.05$. F-test values correspond to different $\mathrm{p}$ values: “*” $=p<0.05$, “**” $=p<0.001$, "n.s" $=p>0.05$. 
Table 5. Foliar nutrient content in maize plants grown in different soil amendments \pm standard error.

\begin{tabular}{|c|c|c|c|c|c|c|c|c|c|c|c|c|c|c|}
\hline \multirow[b]{2}{*}{ Treatment } & \multicolumn{7}{|c|}{$\mathrm{g} / \mathrm{kg}$} & \multicolumn{7}{|c|}{$\mathrm{mg} / \mathrm{kg}$} \\
\hline & $\mathbf{N}$ & $P$ & K & $\mathrm{Ca}$ & $\mathrm{Mg}$ & $\mathrm{Na}$ & $\mathrm{s}$ & $\mathrm{Fe}$ & $\mathrm{Pb}$ & Mn & $\mathrm{Cu}$ & $\mathrm{Zn}$ & $\mathrm{Cr}$ & $\mathrm{Cd}$ \\
\hline C + BSD $1 \%$ & $20.38 \pm 0.63$ & $1.45 \pm 0.10$ & $4.33 \pm 0.16$ & $3.49 \pm 0.08 \mathrm{ab}$ & $1.90 \pm 0.01 \mathrm{~b}$ & $0.95 \pm 0.04 \mathrm{~b}$ & $8.97 \pm 0.06$ & $236.34 \pm 4.15 c$ & $4.83 \pm 0.42$ & $93.11 \pm 1.22 \mathrm{ab}$ & $4.73 \pm 1.25$ & $125.5 \pm 3.02$ & $4.73 \pm 0.10$ & $3.37 \pm 0.45$ \\
\hline & $21.61 \pm 1.55$ & $1.48 \pm 0.08$ & & & & & $7.69 \pm 0.60$ & $273.23 \pm 3.58 b$ & & $96.19 \pm 1.01 \mathrm{a}$ & & & & \\
\hline$C+B$ & $27 \pm 1.43$ & $1.57 \pm 0.16$ & $4.79 \pm 0.11$ & $84 \pm 0.01 \mathrm{a}$ & $1.96 \pm 0.05 \mathrm{ab}$ & $.22 \pm 0.03 a$ & $8.29 \pm 0.47$ & $293.33 \pm 4.85 a$ & $4.84 \pm 0.54$ & $96.33 \pm 0.58 \mathrm{a}$ & $5.20 \pm 0.50$ & $128.4 \pm 4.28$ & $4.15 \pm 0.92$ & $3.38 \pm 0.34$ \\
\hline $\mathrm{C}+\mathrm{SD} 1 \%$ & $18.50 \pm 0.97$ & $1.41 \pm 0.10$ & $4.04 \pm 0.20$ & $2.45 \pm 0.05 \mathrm{~d}$ & $1.64 \pm 0.05 \mathrm{~cd}$ & $1.00 \pm 0.02 b$ & $8.42 \pm 0.42$ & $163.01 \pm 4.80 \mathrm{ef}$ & $4.91 \pm 0.43$ & $88.21 \pm 3.51 \mathrm{a}-\mathrm{c}$ & $3.75 \pm 0.67$ & $122.2 \pm 1.83$ & $3.88 \pm 0.54$ & $3.46 \pm 0.47$ \\
\hline $\mathrm{C}+\mathrm{SD} 2 \%$ & $0.72 \pm 1.45$ & $1.44 \pm 0.15$ & $2 \pm 0.10$ & $2.58 \pm 0$. & & & & $155.66 \pm 2.00 \mathrm{f}$ & $4.37 \pm 0.57$ & & \pm 1.06 & $1 \pm 2.43$ & \pm 0.31 & $3.14 \pm 0.34$ \\
\hline $\mathrm{C}+\mathrm{SD} 3 \%$ & $19.54 \pm 1.70$ & $1.42 \pm 0.17$ & $6 \pm 0.16$ & $2.67 \pm 0$ & & $1.08 \pm 0$ & $6.54 \pm 0.41$ & $158.66 \pm 3$ & $4.63 \pm 0.39$ & $5 \pm 1.55 \mathrm{a}$ & $3.99 \pm 0.16$ & $125.0 \pm 1.75$ & $4.56 \pm 0.62$ & $3.45 \pm 0.48$ \\
\hline$C+B C 1 \%$ & $18.94 \pm 0.91$ & $1.43 \pm 0$ & & & & & & & & & & & & \\
\hline$C+B C 2 \%$ & $19.79 \pm 0.66$ & $1.44 \pm 0.14$ & $4.00 \pm 0$ & $2.86=$ & & & & $195.66 \pm$ & & & & 120.9 & & \\
\hline$C+B C 3 \%$ & $17.87 \pm 1.66$ & $1.46 \pm 0.19$ & 3.99 & $2.64 \pm$ & & & & & $4.30 \pm 0$ & 81. & & & & $3.42 \pm 0.50$ \\
\hline C & $19.73 \pm 1.06$ & $1.52 \pm 0.20$ & $4.08 \pm 0.12$ & $2.07 \pm 0.03 e$ & $1.54 \pm 0.06 \mathrm{~cd}$ & $0.97 \pm 0.04 \mathrm{~b}$ & $8.03 \pm 0.42$ & $112.24 \pm 1.12 \mathrm{~g}$ & $3.79 \pm 0.52$ & $78.23 \pm 3.18 c$ & $3.75 \pm 0.56$ & $117.2 \pm 5.70$ & $4.28 \pm 0.14$ & $3.57 \pm 0.41$ \\
\hline
\end{tabular}

BSD, solid-digestate-derived biochar; SD, solid digestate; BC, wood-derived biochar; $\mathrm{C}$, control. Means followed by the same letters on the column are not significant at $p>0.05$, and means

followed by different letters on the column are significant at $p<0.05$. F-test values correspond to different $\mathrm{p}$ values: "**" $=p<0.05,{ }^{\prime \prime * * \prime \prime}=p<0.001$, “n.s" $=p>0.05$. 


\section{Discussion}

\subsection{Effect of Amendment Application on Soil Properties}

Solid-separate digestate (SD) was confirmed to have a neutral-slightly alkaline pH. However, SD showed a slightly lower $\mathrm{pH}$ value with respect to the range from 7.3 to 8.7 reported in literature, probably due to the different digested feedstock and the adopted process parameters [30,31]. Both tested biochar types were also alkaline, in agreement with Dominugues et al. [18], who observed pH values higher than 8 when using biochar derived from wood and nutrient-rich residues pyrolyzed at a temperature above $450^{\circ} \mathrm{C}$. These data confirmed biochar's ability to correct soil acidity [9]. However, BSD showed a higher liming value compared to that of BC as a result of higher ash content and a large amount of inorganic compounds [4,18].

Concerning EC, higher SD values were reported compared to those observed in other studies ( 2.73 and $5.66 \mathrm{mS} \mathrm{cm}^{-1}$ ), while the tested biochar types were within the range from 0.15 to $8.2 \mathrm{mS} \mathrm{cm}^{-1}$ reported in literature [30,32,33]. However, $\mathrm{pH}$ and EC data recorded in the treatments were not considered a limiting factor for plant, soil-macrofauna, and micro-organism growth [34,35].

Several studies consider biochar VM content as a key parameter to evaluate effective agronomic performance on soil properties and plant growth [18,36,37]. VMs are compounds (such as carboxylic acid, phenol, ketone, and aldehyde functional groups) that are volatilized during a pyrolysis process by simultaneous chemical reactions, or the cracking or rearrangement of lignocellulosic chemical bonds $[13,38]$. These compounds could inhibit root growth or serve as a labile C pool susceptible to degradation by soil micro-organisms; the content seems to be most affected by pyrolysis temperature and less by initial feedstock selection [3,4]. Nevertheless, VM content measured in BC was almost doubled compared to that in BSD, in agreement with results previously reported in literature $[4,6,9,18]$. However, the authors suggested that this parameter does not represent an accurate estimation of biochar stability, especially when biochar is characterized by a high ash fraction that accelerates degradation rates and leads to a shorter lifetime in the soil system [6,39].

In contrast to $\mathrm{VM}$, ash content depends less on pyrolysis temperature than on the content of inorganic compounds (i.e., $\mathrm{P}, \mathrm{K}, \mathrm{Ca}$, and $\mathrm{Mg}$ ) in the feedstock $[4,18]$. As expected, proximate analysis showed a lower ash value in wood-derived biochar in contrast to a higher concentration in pyrolyzed agricultural waste or annual crop residues $[9,40]$. Biochar with a high ash content tends to be appreciated for its nutrient content, but generally shows a lower fixed $C$ value (even if this depends on process condition and reactor configuration), which is closely related to stable carbon content, and thus improves the longevity of the carbon-sequestration tool [36,41]. Indeed, Enders et al. [4] found a negative correlation between ash content and fixed $C$, suggesting that the formation of carbon aromatic forms is hindered by the presence of ash. Biochar characterized by high fixed $C$ is appraised not only to offset GHG emissions due to C-fixing, but also because it requires fewer applications to maintain carbon-sequestration activity and possible positive amendment effects in soil over time $[4,6]$.

Changes in soil nutrient concentration after amendment application vary greatly and depend mainly on amendment selection than on application rate [3,20]. Results confirmed a higher microelement content in biochar using material originating from cellulosic or lignocellulosic feedstocks with respect to those obtained by lignin feedstock [20-22,42]. In fact, BSD was reported to have the highest presence of inorganic elements, likely due to the more nutrient-rich feedstock (i.e., solid digestate) as compared with the raw material (i.e., wood residues) selected to produce BC [9]. At the same time, BSD showed the same trend with respect to the initial material (i.e., SD), mainly due to the concentration of these elements that were not lost by volatilization during pyrolysis [4,18]. However, the contents of investigated macro- and microelements were within the range suggested by the European Biochar Certificate, and recovered heavy-metal concentrations in the tested rates seemed to not be toxic for plant growth or vegetative-parameter development $[43,44]$. 


\subsection{Amendment Influence on Maize Growth}

The amendment application in soil appeared to influence all investigated vegetative parameters, both positive and negative, as previously reported by several authors $[9,45]$

In agreement with previous work by Chan et al. [5], the best performances were found in the early stages of maize growth, when the applied biochar was nutrient-rich-derived (BSD). Similarly, Uzoma et al. [46] reported favorable use of cow-manure biochar in first-year maize cultivation, probably due to the high ion concentrations and relatively quick release compared to that of other plant-derived biochar [20]. This trend was also confirmed by foliar nutrient content, where a high microelement presence in biochar caused greater leaf accumulation and plant development both in maize and geranium for essential-oil (Pelargonium graveolens) cultivation [12,47]. Concerning application rate, the authors reported positive maize growth using biochar up to $2 \%-3 \% \mathrm{w} / \mathrm{w}[13,48]$. Ahmed et al. [49] suggested that exceeding $2 \% \mathrm{w} / \mathrm{w}$ biochar rate in the substrate could cause a decline in cob weight and plant biomass in maize due to biochar ethylene emission, which leads to lost root geotropism. Moreover, Deenik et al. [3] observed an increase in maize-biomass production up to the $5 \% \mathrm{w} / \mathrm{w}$ threshold, and a decrease in plant parameters when biochar application exceeded this rate. This could have been caused by a reduction in plant nutrient uptake when high biochar rates were reached [46].

When wood-derived-biochar (BC) rates were applied, plants were reported to have the worst vegetative and growth performance, probably due to the low nutrient concentrations and high VM content recovered in wood-derived biochar [20,50]. From an agronomic standpoint, biochar characterized by high VM content may have affected the nitrogen biochemical cycling in the soil system by reducing $N$ uptake, thus negatively influencing plant growth [3,37]. For example, Deenik et al. [51] observed significantly better growth in maize using low VM (6.3\%) biochar compared with that with high VM (22.5\%). The authors speculated that the C source of the high VM biochar stimulated immobilization of available plant $\mathrm{N}$ in the soil, causing a reduction in $\mathrm{N}$ uptake by $50 \%$ compared with the control. The same phenomenon was observed by Rondon et al. [52] using biochar produced from the logs of Eucalyptus deglupta with a VM content of 33\%, reporting low $\mathrm{N}$ availability compared to the control and consequently low photosynthate production in Phaseolus vulgaris L. Several studies observed that the alteration of $\mathrm{N}$ dynamics regards both the ammonium $\left(\mathrm{NH}_{4}{ }^{+}\right)$and the nitrate $\left(\mathrm{NO}_{3}{ }^{-}\right)$ form, and magnitude seems to vary according to different thermal-process technologies used during biochar production $[3,53,54]$

In addition, biochar with a high VM content seemed to not be a suitable amendment in the short term [51]. In fact, Jones et al. [55] reported no effect on maize growth in the first year of wood-derived-biochar application. On the other hand, positive results were observed in the subsequent cultivation of Dactylis glomerata. The increasing grass-crop nutritional quality in the second year after biochar application, and the increase in biomass production after the third year, probably occurred due to biochar mineralization for fertilizer application. Indeed, several authors suggested that coapplication of biochar and inorganic $\mathrm{N}$ fertilizer could be a sustainable method to improve crop yield within the year of biochar application $[2,20,50]$.

Moreover, digestate is a nutrient-rich byproduct of the biogas anaerobic-fermentation process that is usually used as fertilizer for supplying nutrients. It can be mechanically separated into liquid $(80 \%-90 \%)$ and solid fractions $(10 \%-20 \%)$ in order to facilitate storage, handling, transport, and distribution [31]. Overall, studies suggested that solid-digestate-fraction application to the soil showed poor short-term fertilization effects due to the relatively slow mineralization rate, which mainly depends on the $\mathrm{NH}_{4}-\mathrm{N}$ pool $[56,57]$. Indeed, by analyzing the growth parameters, SD was confirmed to release available nutrients after about two months of application, providing plant growth compared to the unamended soil [58]. This is a typical phenomenon of amended organic soil that seems to considerably occur in solid-digestate fractions with respect to liquid ones, causing a lack of nutrient synchrony between organic nutrient release and maize nutrient uptake $[59,60]$. 


\section{Conclusions}

Organic-amendment application in soil has developed in recent years, as it is a promising method to enhance sustainable agriculture and climate-change mitigation. In this study, the application of amendments resulted in different effects on soil parameters and crop-yield improvement. In general, all treatments resulted in a $\mathrm{pH}$ and EC increase compared to the soil, while only BSD and SD showed a significant increase in secondary macro- and micronutrient-element content (i.e., $\mathrm{Ca}, \mathrm{Mg}, \mathrm{Na}, \mathrm{Fe}, \mathrm{Mn}$, and $\mathrm{Zn}$ ). At the same time, slow SD nutrient release was reported in all application rates, approximately 60 days after application, while BSD showed the best growth conditions in increasing rates for plant height, stem diameter, biomass dry weight, and seed number and weight in maize cultivation. When BC was used, maize recorded the worst results, often the lowest with respect to the soil control. This could be explained by the low presence of nutrients and the relatively high VM content that could have led to an alteration of $\mathrm{N}$ dynamics.

Indeed, from an agronomic standpoint, the utilization of biochar derived from wood feedstock could be considered as a soil conditioner rather than fertilizer, but it can be a sustainable option for environmental clean-up purposes by mitigating the negative effects of greenhouse gases. At the same time, the application of biochar produced from nutrient-rich feedstock in soil seems to be indicated as both soil amendment and fertilizer. However, considering the wide range of biochar variability, further studies are needed to assess the agronomic value via a long-term experiment on the field scale.

Author Contributions: Conceptualization, A.C. and E.P.; methodology, E.P, A.M.; software, A.M.; validation, A.M., D.C. (David Casini) and A.C.; formal analysis, A.M.; investigation, A.C.; resources, E.P.; data curation, A.C.; writing-original draft preparation, A.C.; writing-review and editing, E.P. and D.C. (David Chiaramonti).; visualization, D.C. (David Casini); supervision, E.P.; project administration, D.C. (David Chiaramonti); funding acquisition, D.C. (David Chiaramonti). All authors have read and agreed to the published version of the manuscript. E.P., Enrico Palchetti; D.C. (David Chiaramonti), David Chiaramonti; A.C., Alessandro Calamai; D.C. (David Casini), David Casini; A.M., Alberto Masoni.

Funding: This research received no external funding.

Conflicts of Interest: The authors declare no conflict of interest.

\section{References}

1. Lehmann, J.; Gaunt, J.; Rondon, M. Bio-char Sequestration in Terrestrial Ecosystems-A Review. Mitig. Adapt. Strateg. Glob. Chang. 2006, 11, 403-427. [CrossRef]

2. Faloye, O.T.; Alatise, M.O.; Ajayi, A.E.; Ewulo, B.S. Synergistic effects of biochar and inorganic fertiliser on maize (zea mays) yield in an alfisol under drip irrigation. Soil Tillage Res. 2017, 174, 214-220. [CrossRef]

3. Deenik, J.L.; McClellan, T.; Uehara, G.; Antal, M.J.; Campbell, S. Charcoal Volatile Matter Content Influences Plant Growth and Soil Nitrogen Transformations. Soil Sci. Soc. Am. J. 2010, 74, 1259-1270. [CrossRef]

4. Enders, A.; Hanley, K.; Whitman, T.; Joseph, S.; Lehmann, J. Characterization of biochars to evaluate recalcitrance and agronomic performance. Bioresour. Technol. 2012, 114, 644-653. [CrossRef]

5. Chan, K.Y.; Van Zwieten, B.L.; Meszaros, I.; Downie, C.A.; Joseph, S. Using poultry litter biochars as soil amendments. Aust. J. Soil Res. 2008, 46, 437-444. [CrossRef]

6. Yargicoglu, E.N.; Sadasivam, B.Y.; Reddy, K.R.; Spokas, K. Physical and chemical characterization of waste wood derived biochars. Waste Manag. 2015, 36, 256-268. [CrossRef] [PubMed]

7. Roy, P.; Dias, G. Prospects for pyrolysis technologies in the bioenergy sector: A review. Renew. Sustain. Energy Rev. 2017, 77, 59-69. [CrossRef]

8. Zhang, M.; Ok, Y.S. Biochar soil amendment for sustainable agriculture with carbon and contaminant sequestration. Carbon Manag. 2014, 5, 255-257. [CrossRef]

9. Spokas, K.A.; Cantrell, K.B.; Novak, J.M.; Archer, D.W.; Ippolito, J.A.; Collins, H.P.; Boateng, A.A.; Lima, I.M.; Lamb, M.C.; McAloon, A.J.; et al. Biochar: A Synthesis of its Agronomic Impact beyond Carbon Sequestration. J. Environ. Qual. 2012, 41, 973. [CrossRef]

10. Inyang, M.; Gao, B.; Ding, W.; Pullammanappallil, P.; Zimmerman, A.R.; Cao, X. Enhanced Lead Sorption by Biochar Derived from Anaerobically Digested Sugarcane Bagasse. Sep. Sci. Technol. 2011, 46, 1950-1956. [CrossRef] 
11. Upadhyay, K.P.; George, D.; Swift, R.S.; Galea, V. The Influence of Biochar on Growth of Lettuce and Potato. J. Integr. Agric. 2014, 13, 541-546. [CrossRef]

12. Calamai, A.; Palchetti, E.; Masoni, A.; Marini, L.; Chiaramonti, D.; Dibari, C.; Brilli, L. The Influence of Biochar and Solid Digestate on Rose-Scented Geranium (Pelargonium graveolens L'Hér.) Productivity and Essential Oil Quality. Agronomy 2019, 9, 260. [CrossRef]

13. Butnan, S.; Deenik, J.L.; Toomsan, B.; Antal, M.J.; Vityakon, P. Biochar characteristics and application rates affecting corn growth and properties of soils contrasting in texture and mineralogy. Geoderma 2015, 237-238, 105-116. [CrossRef]

14. Nelissen, V.; Ruysschaert, G.; Manka'Abusi, D.; D’Hose, T.; De Beuf, K.; Al-Barri, B.; Cornelis, W.; Boeckx, P. Impact of a woody biochar on properties of a sandy loam soil and spring barley during a two-year field experiment. Eur. J. Agron. 2015, 62, 65-78. [CrossRef]

15. Crane-Droesch, A.; Abiven, S.; Jeffery, S.; Torn, M.S. Heterogeneous global crop yield response to biochar: A meta-regression analysis. Environ. Res. Lett. 2013, 8, 044049. [CrossRef]

16. Joseph, S.D.; Camps-Arbestain, M.; Lin, Y.; Munroe, P.; Chia, C.H.; Hook, J.; Van Zwieten, L.; Kimber, S.; Cowie, A.; Singh, B.P.; et al. An investigation into the reactions of biochar in soil. Aust. J. Soil Res. 2010, 48, 501-515. [CrossRef]

17. Rogovska, N.; Laird, D.; Cruse, R.M.; Trabue, S.; Heaton, E. Germination Tests for Assessing Biochar Quality. J. Environ. Qual. 2012, 41, 1014. [CrossRef]

18. Domingues, R.R.; Trugilho, P.F.; Silva, C.A.; Melo, I.C.N.A.; de Melo, L.C.A.; Magriotis, Z.M.; Sánchez-Monedero, M.A. Properties of biochar derived from wood and high-nutrient biomasses with the aim of agronomic and environmental benefits. PLoS ONE 2017, 12, e0176884. [CrossRef]

19. Gul, S.; Whalen, J.K.; Thomas, B.W.; Sachdeva, V.; Deng, H. Physico-chemical properties and microbial responses in biochar-amended soils: Mechanisms and future directions. Agric. Ecosyst. Environ. 2015, 206, 46-59. [CrossRef]

20. Scott, H.; Ponsonby, D.; Atkinson, C.J. Biochar: An improver of nutrient and soil water availability-What is the evidence? CAB Rev. Perspect. Agric. Vet. Sci. Nutr. Nat. Resour. 2014, 9, 1-19. [CrossRef]

21. Monlau, F.; Sambusiti, C.; Ficara, E.; Aboulkas, A.; Barakat, A.; Carrère, H. New opportunities for agricultural digestate valorization: Current situation and perspectives. Energy Environ. Sci. 2015, 8, 2600-2621. [CrossRef]

22. Yuan, P.; Wang, J.; Pan, Y.; Shen, B.; Wu, C. Review of biochar for the management of contaminated soil: Preparation, application and prospect. Sci. Total Environ. 2019, 659, 473-490. [CrossRef] [PubMed]

23. Rizzo, A.M.; Pettorali, M.; Nistri, R.; Chiaramonti, D. Mass and energy balances of an autothermal pilot carbonization unit. Biomass Bioenergy 2019, 120, 144-155. [CrossRef]

24. Olsen, S.R.; Sommers, L.E. Phosphorus, Methods of Soil Analysis, 2nd ed.; ASA and SSSA: Madison, WI, USA, 1982.

25. Ahmedna, M.; Marshall, W.; Rao, R. Production of granular activated carbons from select agricultural by-products and evaluation of their physical, chemical and adsorption properties. Bioresour. Technol. 2000, 71, 113-123. [CrossRef]

26. R Development Core Team. R: A Language and Environment for Statistical Computing; R Foundation for Statistical Computing: Vienna, Austria, 2013.

27. Bates, D.; Mächler, M.; Bolker, B.; Walker, S. Fitting Linear Mixed-Effects Models Using lme4. J. Stat. Softw. 2015, 67, 1-48. [CrossRef]

28. Hothorn, T.; Bretz, F.; Westfall, P.; Heiberger, R.M.; Schuetzenmeister, A.; Scheibe, S.; Hothorn, M.T. Package 'Multcomp'. Available online: http://cran.stat.sfu.ca/web/packages/multcomp/multcomp (accessed on 10 November 2019).

29. Hothorn, T.; Bretz, F.; Westfall, P. Simultaneous Inference in General Parametric Models. Biom. J. 2008, 50, 346-363. [CrossRef] [PubMed]

30. Stefaniuk, M.; Oleszczuk, P. Characterization of biochars produced from residues from biogas production. J. Anal. Appl. Pyrolysis 2015, 115, 157-165. [CrossRef]

31. Palchetti, E.; Calamai, A.; Verdi, L.; Masoni, A.; Marini, L.; Chiaramonti, D. Preliminary screening of agricultural feedstocks for anaerobic digestion. Adv. Hortic. Sci. 2019, 33, 333-344. [CrossRef]

32. Wiedner, K.; Rumpel, C.; Steiner, C.; Pozzi, A.; Maas, R.; Glaser, B. Chemical evaluation of chars produced by thermochemical conversion (gasification, pyrolysis and hydrothermal carbonization) of agro-industrial biomass on a commercial scale. Biomass Bioenergy 2013, 59, 264-278. [CrossRef] 
33. Pituello, C.; Francioso, O.; Simonetti, G.; Pisi, A.; Torreggiani, A.; Berti, A.; Morari, F. Characterization of chemical-physical, structural and morphological properties of biochars from biowastes produced at different temperatures. J. Soils Sediments 2015, 15, 792-804. [CrossRef]

34. Yan, N.; Marschner, P.; Cao, W.; Zuo, C.; Qin, W. Influence of salinity and water content on soil microorganisms. Int. Soil Water Conserv. Res. 2015, 3, 316-323. [CrossRef]

35. Chong, C.; Rinker, D.L. Use of Spent Mushroom Substrate for Growing Containerized Woody Ornamentals: An Overview. Compost Sci. Util. 1994, 2, 45-53. [CrossRef]

36. Zimmerman, A.R. Abiotic and Microbial Oxidation of Laboratory-Produced Black Carbon (Biochar). Environ. Sci. Technol. 2010, 44, 1295-1301. [CrossRef]

37. Gundale, M.J.; DeLuca, T.H. Charcoal effects on soil solution chemistry and growth of Koeleria macrantha in the ponderosa pine/Douglas-fir ecosystem. Biol. Fertil. Soils 2006, 43, 303-311. [CrossRef]

38. Bourke, J.; Manley-Harris, M.; Fushimi, C.; Dowaki, K.; Nunoura, T.; Antal, M.J. Do All Carbonized Charcoals Have the Same Chemical Structure? A Model of the Chemical Structure of Carbonized Charcoal. Ind. Eng. Chem. Res. 2007, 46, 5954-5967. [CrossRef]

39. Huisman, D.J.; Braadbaart, F.; van Wijk, I.M.; van Os, B.J.H. Ashes to ashes, charcoal to dust: Micromorphological evidence for ash-induced disintegration of charcoal in Early Neolithic (LBK) soil features in Elsloo (The Netherlands). J. Archaeol. Sci. 2012, 39, 994-1004. [CrossRef]

40. Antal, J.; Grønli, M. The Art, Science, and Technology of Charcoal Production. Am. Chem. Soc. 2003, 42, 1619-1640. [CrossRef]

41. Crombie, K.; Mašek, O.; Sohi, S.P.; Brownsort, P.; Cross, A. The effect of pyrolysis conditions on biochar stability as determined by three methods. GCB Bioenergy 2013, 5, 122-131. [CrossRef]

42. Raveendran, K.; Ganesh, A.; Khilar, K.C. Influence of mineral matter on biomass pyrolysis characteristics. Fuel 1995, 74, 1812-1822. [CrossRef]

43. European Biochar Certificate. Guidelines for Biochar Production; Ithaka Institute: Arbaz, Switzerland, 2012; Volume 4.2.

44. Khan, S.; Waqas, M.; Ding, F.; Shamshad, I.; Arp, H.P.H.; Li, G. The influence of various biochars on the bioaccessibility and bioaccumulation of PAHs and potentially toxic elements to turnips (Brassica rapa L.). J. Hazard. Mater. 2015, 300, 243-253. [CrossRef]

45. Karer, J.; Wimmer, B.; Zehetner, F.; Kloss, S.; Soja, G. Biochar application to temperate soils: Effects on nutrient uptake and crop yield under field conditions. Agric. Food Sci. 2013, 22, 390-403. [CrossRef]

46. Uzoma, K.C.; Inoue, M.; Andry, H.; Fujimaki, H.; Zahoor, A.; Nishihara, E. Effect of cow manure biochar on maize productivity under sandy soil condition. Soil Use Manag. 2011, 27, 205-212. [CrossRef]

47. Awad, Y.M.; Lee, S.-E.; Ahmed, M.B.M.; Vu, N.T.; Farooq, M.; Kim, I.S.; Kim, H.S.; Vithanage, M.; Usman, A.R.A.; Al-Wabel, M.; et al. Biochar, a potential hydroponic growth substrate, enhances the nutritional status and growth of leafy vegetables. J. Clean. Prod. 2017, 156, 581-588. [CrossRef]

48. Haider, G.; Koyro, H.W.; Azam, F.; Steffens, D.; Müller, C.; Kammann, C. Biochar but not humic acid product amendment affected maize yields via improving plant-soil moisture relations. Plant Soil 2014, 395, 141-157. [CrossRef]

49. Ahmed, F.; Arthur, E.; Plauborg, F.; Andersen, M.N. Biochar Effects on Maize Physiology and Water Capacity of Sandy Subsoil. Mech. Agric. Conserv. Resour. 2016, 6, 8-13.

50. Partey, S.T.; Preziosi, R.F.; Robson, G.D. Short-Term Interactive Effects of Biochar, Green Manure, and Inorganic Fertilizer on Soil Properties and Agronomic Characteristics of Maize. Agric. Res. 2014, 3, 128-136. [CrossRef]

51. Deenik, J.; Mcclellan, A.T.; Uehara, G. Biochar volatile matter content effects on plant growth and nitrogen transformations in a tropical soil. In Proceedings of the Western Nutrient Management Conference, Salt Lake City, UT, USA, 4-5 March 2009; Volume 8, pp. 26-39.

52. Rondon, M.; Lehmann, J.; Ramírez, J.; Hurtado, M. Biological nitrogen fixation by common beans (Phaseolus vulgaris L.) increases with biochar additions. Biol. Fert. Soils 2007, 43, 688-708. [CrossRef]

53. Knowles, O.A.; Robinson, B.H.; Contangelo, A.; Clucas, L. Biochar for the mitigation of nitrate leaching from soil amended with biosolids. Sci. Total Environ. 2011, 409, 3206-3210. [CrossRef]

54. Clough, T.J.; Bertram, J.E.; Ray, J.L.; Condron, L.M.; O'Callaghan, M.; Sherlock, R.R.; Wells, N.S. Unweathered Wood Biochar Impact on Nitrous Oxide Emissions from a Bovine-Urine-Amended Pasture Soil. Soil Sci. Soc. Am. J. 2010, 74, 852. [CrossRef] 
55. Jones, D.L.; Rousk, J.; Edwards-Jones, G.; DeLuca, T.H.; Murphy, D.V. Biochar-mediated changes in soil quality and plant growth in a three year field trial. Soil Biol. Biochem. 2012, 45, 113-124. [CrossRef]

56. de Boer, H.C. Co-digestion of Animal Slurry Can Increase Short-Term Nitrogen Recovery by Crops. J. Environ. Qual. 2008, 37, 1968-1973. [CrossRef] [PubMed]

57. Laboski, C.A.M.; Earhart, S.M.; Baxter, C.A. Evaluation of nitrogen availability from raw and treated dairy manures. In Proceedings of the 19th World Congress of Soil Science, Soil solutions for a Changing World, Brisbane, Australia, 1-6 August 2010.

58. Grigatti, M.; Cavani, L.; Marzadori, C. Recycling of Dry-Batch Digestate as Amendment: Soil C and N Dynamics and Ryegrass Nitrogen Utilization Efficiency. Waste Biomass Valorization 2014, 5, 823. [CrossRef]

59. Gutser, R.; Ebertseder, T.; Weber, A.; Schraml, M.; Schmidhalter, U. Short-term and residual availability of nitrogen after long-term application of organic fertilizers on arable land Summary-Zusammenfassung. J. Plant Nutr. Soil Sci. 2005, 168, 439-446. [CrossRef]

60. Maucieri, C.; Nicoletto, C.; Caruso, C.; Sambo, P.; Borin, M. Effects of digestate solid fraction fertilisation on yield and soil carbon dioxide emission in a horticulture succession. Ital. J. Agron. 2017, 11, 116-123. [CrossRef]

(C) 2020 by the authors. Licensee MDPI, Basel, Switzerland. This article is an open access article distributed under the terms and conditions of the Creative Commons Attribution (CC BY) license (http://creativecommons.org/licenses/by/4.0/). 